\title{
CONTINUOUS MONITORING OF CIRCULATING BLOOD HEMATOCRIT
}

\author{
Yoshifumi TanaKa, Taketoshi Morimoto, Hiroshi Watari, \\ and Masao MrYazAKI \\ Department of Anesthesiology and Department of Physiology, \\ Kyoto Prefectural University of Medicine, \\ Kyoto 602, Japan
}

\begin{abstract}
The relationship between the hematocrit value and the absolute value of impedance of whole blood was analyzed. The results indicated that the hematocrit value was linearly proportional to the logarithm of the impedance of whole blood over the range of hematocrit values of 10 to $60 \%$ with a correlation coefficient of 0.995 . The circuit used for detection of the impedance of whole blood was described. It required no adjustments during measurements and had a wide linear range. The sources of error during continuous measurements were the rate of blood flow through the sensor cell, the temperature of the blood, and the concentrations of plasma proteins and ions. Accordingly, the flow and temperature of blood were kept constant during measurements. Changes in the concentrations of plasma proteins and ions within physiological ranges caused errors of $\pm 2.0 \%$. Typical results of continuous measurements were presented, showing that the hematocrit values obtained by this method agreed well with those obtained by the conventional method from the packed cell volume. An advantage of this method over the conventional method was that it could follow rapid and transient changes in the hematocrit.
\end{abstract}

The hematocrit, i.e., the volume of erythrocytes as a percentage of the whole blood, is an important index of hemoconcentration or hemodilution. The standard method for determining the hematocrit value is to centrifuge blood samples and measure the packed cell volume (Packed Cell Volume Method). This method cannot be used to monitor rapid and transient changes in the hematocrit because time is required for blood sampling and centrifugation. Another method of measuring hematocrit values from the conductivity of whole blood (Cell Conductivity Volume Method) has been used for field work (KERNER et al., 1961) and autoanalyzers

Received for publication January 7, 1976

田中義文，森本武利，亘 弘，宮崎正夫 
(DAvis et al., 1966). Values obtained by this method were empirically calibrated against those obtained by the Packed Cell Volume Method. In this work, we studied the application of the Cell Conductivity Volume Method for continuous monitoring of the hematocrit of circulating blood. The relationship between the hematocrit and the impedance of the blood and sources of error were analyzed.

\section{METHODS}

Instrument. The circuit used for measurement of the impedance of blood is shown in Fig. 1. Its output is proportional to the absolute value of the impedance

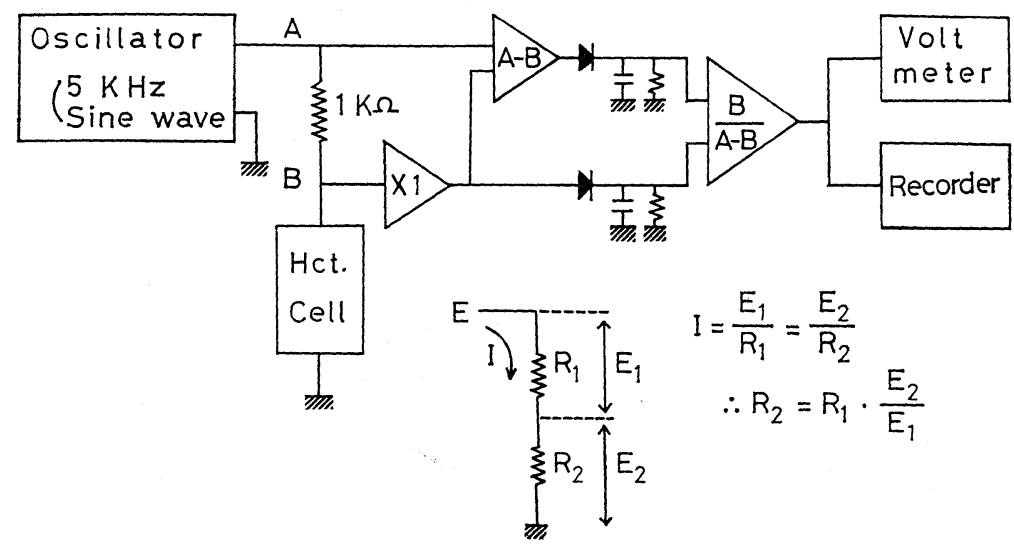

Fig. 1. Circuit diagram of the hematocrit meter. For details, see text.

of the blood sample in the hematocrit cell. The operation of the circuit is shown schematically in the figure. $\quad R_{1}$ is a resistance of $1 \mathrm{k} \Omega$ and $R_{2}$ is the resistance of the hematocrit cell. The currents through $R_{1}$ and $R_{2}$ should be equal and are $E_{1} / R_{1}$ and $E_{2} / R_{2}$, respectively. Accordingly, the resistance of the hematocrit cell $R_{2}$ (in $\mathrm{k} \Omega$ ) must be $\mathrm{E}_{2} / \mathrm{E}_{1}$. To obtain $\mathrm{E}_{1}$ (the voltage difference between $\mathrm{A}$ and $\mathrm{B}$ in Fig. 1), the voltage at point $B$ is substracted from that at point $A$ by means of an operation amplifier (Analog Devices 741 ). $\quad E_{2}$, the voltage at point $B$, and $E_{1}$ are converted to direct current. The linearity of the A-D convertion is maintained choosing $R_{1}$ within the same order with $R_{2}$. The ratio of $E_{2} / E_{1}$ is obtained by a divider operation amplifier (Analog Devices 433J).

The characteristics of the circuit are as follows: The output reading is very stable irrespective of fluctuation of the oscillator output voltage, no balancing of the circuit is necessary and the linear range is wider than that in the bridge method conventionally used for impedance measurements (OKADA and SCHWAN, 1960; Rothe et al., 1967). The hematocrit cell is composed of a plexiglass tube of $3 \mathrm{~mm}$ inner diameter with two platinum electrodes of $0.1 \mathrm{~cm}^{2}$ surface area fixed to the 
inner surface. The tube is connected to an extra-corporeal circulation for animal experiments.

Animal experiments. Six mongrel dogs were used. They were anesthetized with i. v. injection of sodium pentobarbital $(30 \mathrm{mg} / \mathrm{kg}$ of body weight) and then respiration was controlled with a Harvard-type respirator and anesthesia was maintained with $1 \%$ Halothane. The femoral arteries and veins on both sides were exposed and an arterio-venous by-pass was made with polyethylene catheters on the right side. In this by-pass, a peristaltic pump (Sigma-motor pump), a temperature controller and the hematocrit cell were connected in series. The arterial and central venous pressures were recorded from the left femoral artery and vein with pressure transducers (Statham P23AA and P23BB). The antecubital ein was also cannulated for infusion of saline solution (Fig. 2). The animals

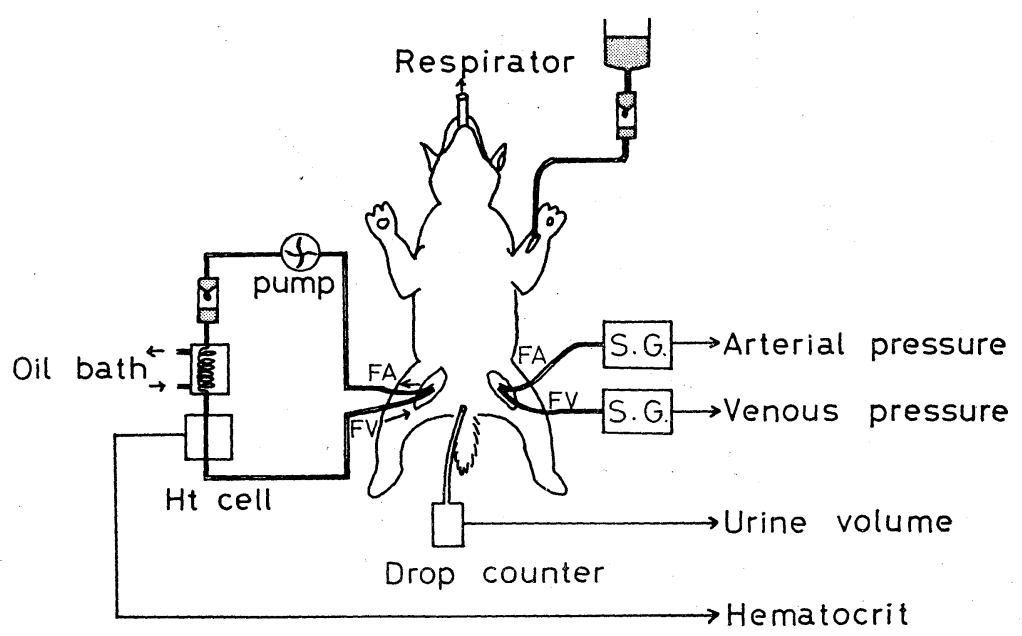

Fig. 2. Schematic representation of the experimental setup for continuous recording of the hematocrit in circulating blood.

were heparinized with an initial dose of 200 units $/ \mathrm{kg}$ and a maintenance dose of 40 units $/ \mathrm{kg} \cdot \mathrm{hr}$ throughout the experiment. The data obtained were analyzed on line with a computer (Hitac-10) and the mean values of the variables measured were typed out every $30 \mathrm{sec}$.

\section{RESULTS AND DISCUSSION}

The relationship between the hematocrit and the electrical impedance of the whole blood is not linear and a Maxwell-Fricke expression has been used for fitting the data (GEDDES and SADLER, 1973). However, for this expression, the impedance of plasma and a shape factor should be determined. Therefore, a simulation study 
was performed to obtain simple relationship between the hematocrit and the electrical impedance.

\section{Calculation of impedance of whole blood}

FRICKE and MORSE (1925) reported that the equivalent circuit shown in Fig. 3 gave a reasonable agreement between the calculated value and the observed value

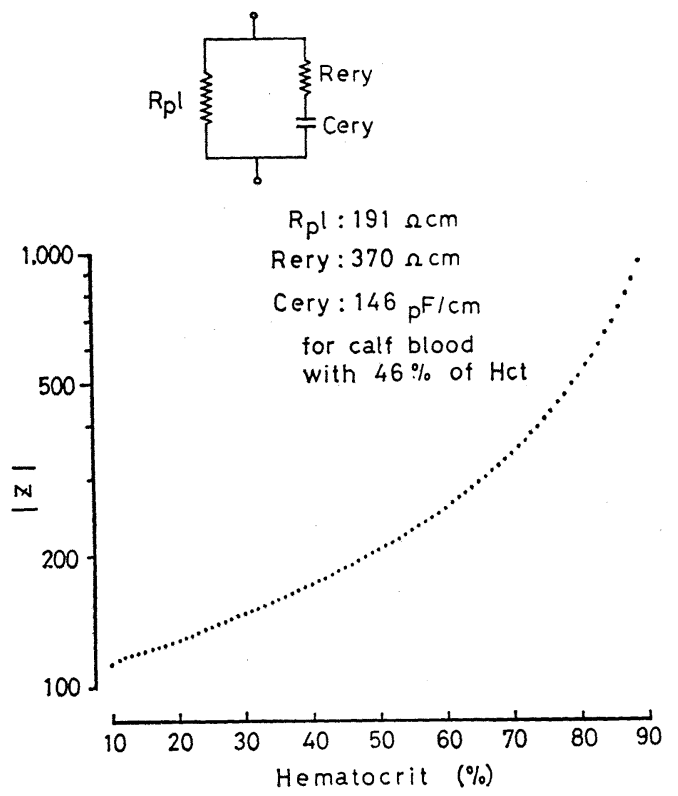

Fig. 3. Calculation of impedance of the blood at various hematocrit values. The equivalent circuit and values used are shown in the upper portion of the figure.

of impedance for erythrocyte suspensions. This circuit was used for the simulation and the following assumptions were made:

$$
\begin{aligned}
& R_{\mathrm{p} 1}=R_{\mathrm{p} 1}^{\prime} \times \frac{1}{1-X} \\
& R_{\text {ery }}=R_{\text {ery }}^{\prime} \times \frac{1}{X} \\
& C_{\text {ery }}=C_{\text {ery }}^{\prime} \times X
\end{aligned}
$$

where $R_{\mathrm{p} 1}$ is the resistance of plasma in the erythrocyte suspension, $R_{\mathrm{p} 1}^{\prime}$ is the resistance of plasma at a hematocrit of $0 \%, R_{\text {ery }}$ is the resistance of erythrocytes in the erythrocyte suspension, $R_{\text {ery }}^{\prime}$ is the resistance of erythrocytes at a hematocrit of $100 \%, C_{\text {ery }}$ is the capacity of erythrocytes in the erythrocyte suspension, $C_{\text {ery }}^{\prime}$ is the capacity of erythrocytes at a hematocrit of $100 \%$ and $X$ represents hemato- 
crit/100. FrICKE and MorSE (1925) reported values of $R_{\mathrm{p} 1}, R_{\text {ery }}$, and $C_{\text {ery }}$ of 191 $\Omega \mathrm{cm}, 350 \Omega \mathrm{cm}$ and $146 \mathrm{pF} \cdot \mathrm{cm}^{-1}$ for calf whole blood with a hematocrit of $46 \%$. Using these values $R_{\mathrm{p} 1}^{\prime}, R_{\mathrm{ery}}^{\prime}$ and $C_{\text {ery }}^{\prime}$ were calculated as $103 \Omega \mathrm{cm}, 170 \Omega \mathrm{cm}$ and $317 \mathrm{pF} \cdot \mathrm{cm}^{-1}$, respectively. The absolute value of impedance $|Z|$ in the equivalent circuit in Fig. 3 can be calculated as follows:

$$
\begin{aligned}
& Z=\frac{1}{\frac{1}{R_{\mathrm{p} 1}}+\frac{1}{R_{\text {ery }}+\frac{1}{j \omega C_{\text {ery }}}}} \\
& |Z|=\frac{R_{\mathrm{p} 1} \sqrt{\left\{1+\omega^{2} C_{\text {ery }}^{2} R_{\text {ery }}\left(R_{\text {ery }}+R_{\mathrm{p} 1}\right)^{2}\right\}^{2}+\omega^{2} C_{\text {erg }}{ }^{2} R_{\mathrm{p} 1}{ }^{2}}}{1+\omega^{2} C_{\text {erg }}^{2}\left(R_{\mathrm{p} 1}+R_{\text {ery }}\right)^{2}}
\end{aligned}
$$

Values of $R_{\mathrm{p} 1}, R_{\mathrm{ery}}$, and $C_{\mathrm{ery}}$ were calculated at hematocrit values of 0 to $99 \%$, and values of $|Z|$ were calculated at each hematocrit value using frequency of $5 \mathrm{kHz}$ $(\omega=2 \pi \times 5)$. In Fig. 3, $\log |Z|$ is plotted against the hematocrit of 10 to $90 \%$. The relation is not linear, but a linear regression with a correlation coefficient of 0.995 is obtained over the range of hematocrit values of 0 to $60 \%$. GEDDES and SADLER (1973) found empirically that the relation between the hematocrit and the resistivity of whole blood was almost similar among human, canine, bovine and equine species. Based on these findings, the linear regression between the hematocrit and $\log |Z|$ was used in the following analysis. A frequency of $5 \mathrm{kHz}$ was used, because the effects of polarization and the capacity of erythrocyte were almost negligible at this frequency.

\section{In vitro calibration and sourses of error}

Figure 4 shows the relationship between the hematocrit and the impedance of the blood. Blood samples were obtained from four dogs, and each sample was centrifuged to separate the cells from the plasma. The cell-plasma mixtures with various hematocrit values were then prepared from the blood of each dog. The output of the volt meter, which is proportional to the impedance of the blood in the hematocrit cell, is plotted on a log scale against the hematocrit value determined by the Packed Cell Volume Method. As shown in the figure, a high correlation was obtained between the two variables, suggesting that the hematocrit can be obtained in this way with a maximum error of $\pm 2 \%$, including individual variation.

Differences in protein and ion concentrations in the plasma have been suggested as sources of this individual variation (KERNER et al., 1961), but no quantitative analysis of the error has been reported. Accordingly, the effects of changes in protein and ion concentrations were determined experimentally. An erythrocyte suspension of a fixed hematocrit $(40 \%)$ was prepared in Ringer's solution and its protein concentration was varied from $2.0 \mathrm{~g} \%$ to $10.0 \mathrm{~g} \%$ by adding powdered albumin. The regression line between $\log |Z|$ of the solution and the protein con- 
centration was $\log |Z|=0.004 \times($ protein concentration in $\mathrm{g} \%)+2.270$, while the regression line obtained between $\log |Z|$ and the hematocrit value at a constant protein concentration of $3.1 \mathrm{~g} \%$ was $\log |Z|=0.008 \mathrm{Ht}+1.920$. Thus $1 \mathrm{~g} \%$

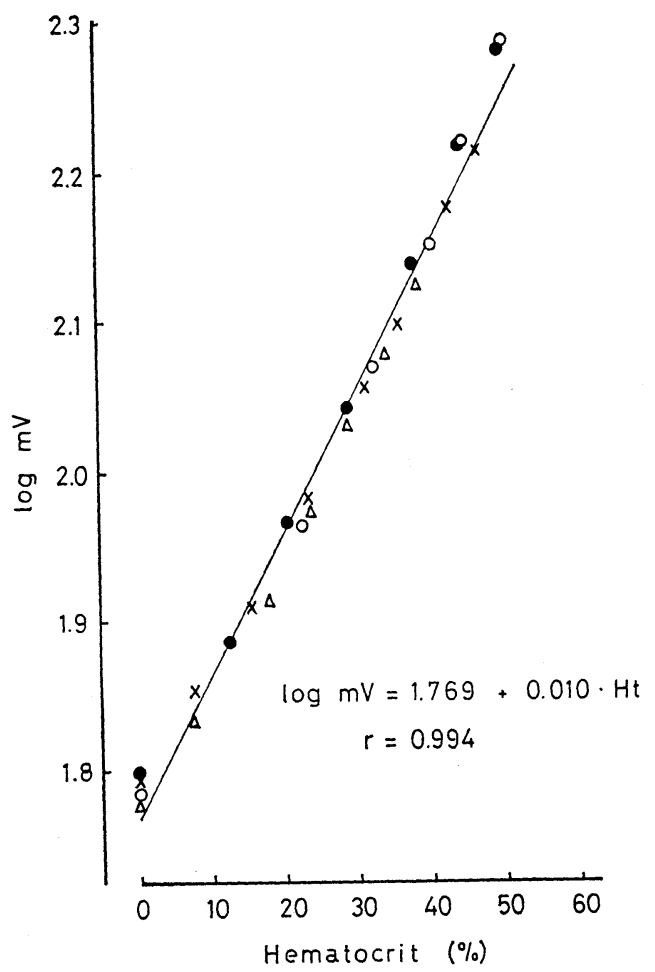

Fig. 4. Hematocrit-impedance relation. The four symbols indicate values for samples from four different dogs. The hematocrit on the abscissa was determined by the conventional Packed Cell Volume Method.

increase in protein concentration caused an increase in volt meter reading equivalent to an increase in the hematocrit of $0.5 \%$. In other words, an increase in the plasma protein concentration of $4 \mathrm{~g} \%$ caused increase in the reading equivalent to $2.0 \%$ increase in the hematocrit. A similar experiment was performed on the effect of change in sodium chloride concentration over the range of 116 to 140 $\mathrm{mEq} / \mathrm{l}$. The results indicated that increase in the sodium chloride concentration of $1 \mathrm{mEq} / 1$ caused decrease in the reading equivalent to $0.2 \%$ decrease in hematocrit. An increase in the sodium chloride concentration of $10 \mathrm{mEq} / 1$ caused decrease in the hematocrit reading of $2.0 \%$. Thus provided that the changes in protein and sodium chloride are within the physiological range, they cause an error of within $\pm 2.0 \%$.

To apply this method to continuous monitoring of circulating blood, the flow 


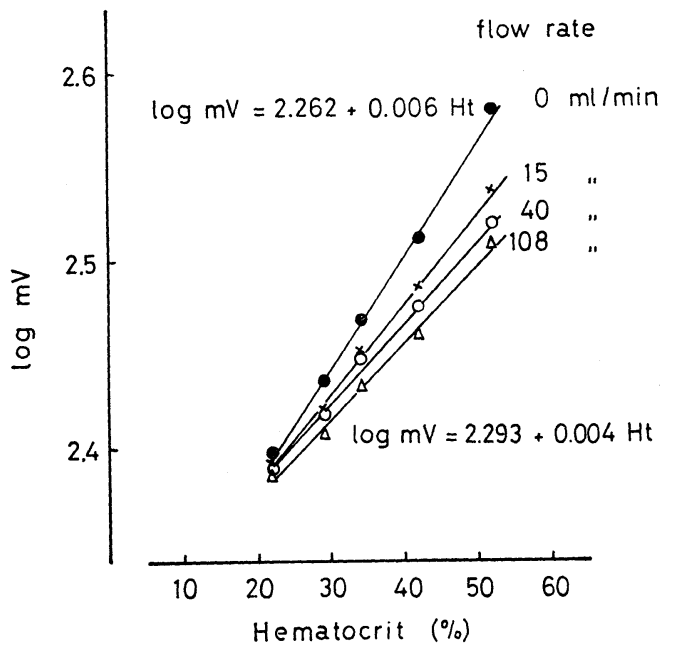

Fig. 5. Effect of the rate of flow on the hematocrit-impedance relation. The hematocrit on the abscissa was determined by the conventional Packed Cell Volume Method.

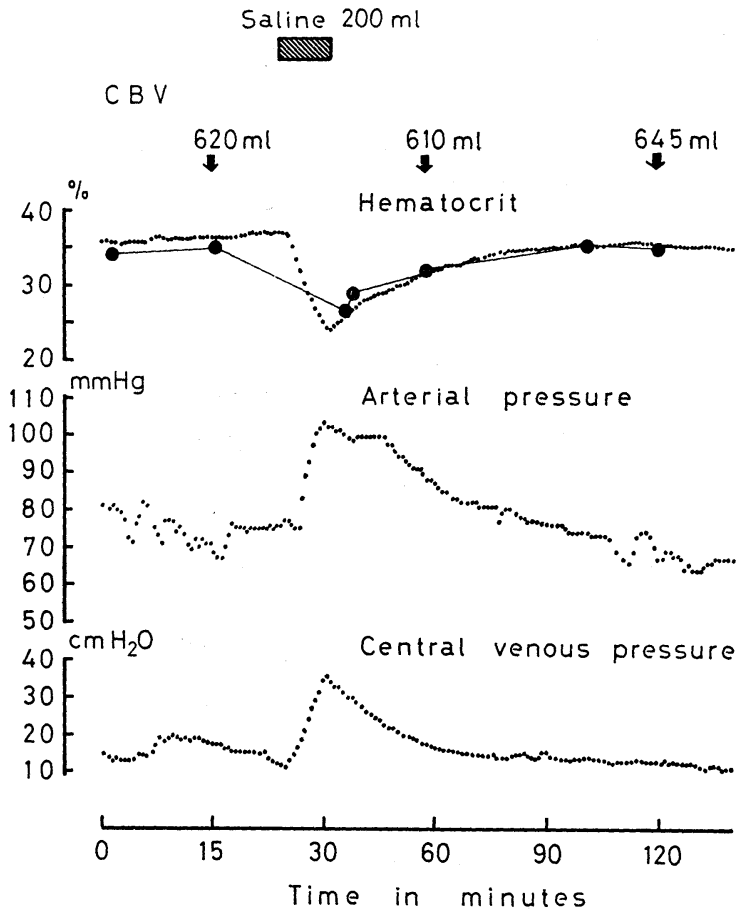

Fig. 6. Continuous measurements of the hematocrit of circulating blood during saline infusion. : hematocrit values obtained by the conventional method. 
rate and the temperature of the blood flowing through the hematocrit cell should be considered. To test this, blood samples of five different hematocrit values were measured at 12 different flow rates of 0 to $108 \mathrm{ml} / \mathrm{min}$. Regression lines obtained at constant flow rates are shown in Fig. 5. As shown in the figure, the regression line becomes less steep as the flow rate increases. The decrease in the slope is prominent up to a flow rate of $15 \mathrm{ml} / \mathrm{min}$ and it becomes very slight at higher flow rates. Based on this result, continuous measurements were performed in vivo at a constant flow rate of $40 \mathrm{ml} / \mathrm{min}$. The output of the meter was also measured over a temperature range of 20 to $40^{\circ} \mathrm{C}$ on the same sample and a regression line of $\log |Z|=-0.029 t+1.735$ was obtained with a correlation coefficient of 0.995 , in which $t$ represented temperature in centigrade. Accordingly, the temperature of blood samples was also controlled during measurements.

\section{Measurements in vivo}

Figure 6 shows typical recordings obtained with this system. The plots recorded every $30 \mathrm{sec}$ are, from top to bottom, the hematocrit, the arterial pressure

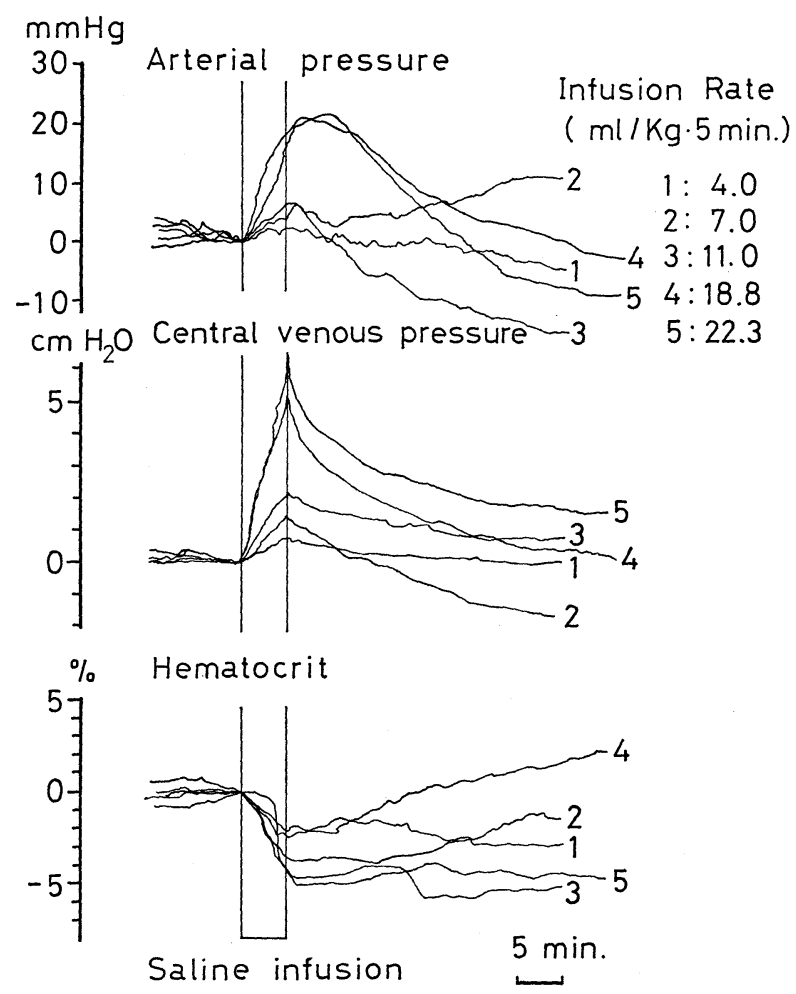

Fig. 7. Changes in hematocrit and blood pressure during saline infusion. Note that changes in the hematocrit are dependent not only on the rate of saline infusion but also on changes in arterial and central venous pressure. 
and the central venous pressure. Circulating blood volumes measured by the ${ }^{131}$ I-RIHSA dilution method are indicated with arrowheads. Closed circles in the hematocrit recording are hematocrit values determined by the conventional Packed Cell Volume Method. The hematocrit values determined from the electrical impedance agreed well with those obtained by the conventional method. The impedance method can detect transient and rapid changes in the hematocrit, as shown in Fig. 7. So far, the hematocrit has mainly been used to measure states of equilibrium, but using the Conductive Cell Volume Method, dynamic changes in the hematocrit can be monitored together with other physiological parameters.

\section{REFERENCES}

Davis, R. E., Bresland, R., and GREe, R. (1966) Evaluation of electronically measured hematocrit values. Lab. Pract., 15: 1376-1378.

FRICKE, H. and MORSE, S. (1925) The electric resistance and capacity of blood for frequencies between 800 and 4 1/2 million cycles. J. Gen. Physiol., 9: 153-167.

GedDES, L. A. and SADLER, C. (1973) The specific resistance of blood at body temperature. Med. Biol. Eng., 11: 336-339.

Kerner, J. A., WURzel, H., and OKadA, R. H. (1961) New electronic method for measuring hematocrit: Clinical evaluation. J. Lab. Clin. Med., 57: 635-641.

OKADA, R. H. and SchWAN, H. P. (1960) An electrical method to determine hematocrits. IRE Trans. Med. Electronics, $7:$ 188-192.

Rothe, C. F., Johnson, J. A., and Moore, W. W. (1967) Continuous measurement of conductivity of biological fluids. J. Appl. Physiol., 23: 1010-1013. 